Pelham, T. G., Hilton, G. S., Railton, C. J., \& Lewis, R. (2015). FDTD model performance analysis for a Cavity Slot Antenna array in a variable geometry conformal test rig. In 2015 IEEE International Symposium on Antennas and Propagation \& USNC/URSI National Radio Science Meeting: Proceedings of a meeting held 19-24 July 2015, Vancouver, British Columbia, Canada (pp. 1836-1837). (Proceedings of IEEE International Symposium on Antennas and Propagation \& USNC/URSI National Radio Science Meeting ). Institute of Electrical and Electronics Engineers (IEEE). https://doi.org/10.1109/APS.2015.7305307

Link to published version (if available):

10.1109/APS.2015.7305307

Link to publication record in Explore Bristol Research

PDF-document

University of Bristol - Explore Bristol Research

General rights

This document is made available in accordance with publisher policies. Please cite only the published version using the reference above. Full terms of use are available:

http://www.bristol.ac.uk/red/research-policy/pure/user-guides/ebr-terms/ 


\section{FDTD Model Performance Analysis for a Cavity Slot Antenna Array in a Variable Geometry Conformal Test Rig}

\author{
Timothy George Pelham \\ Center for Doctoral Training in Communications \\ University of Bristol \\ Bristol, UK \\ Email: t.g.pelham@bristol.ac.uk
}

\author{
Geoff Hilton \\ University of Bristol \\ Bristol, UK
Christopher Railton
University of Bristol \\ Bristol, UK
}

\author{
Rob Lewis \\ BAE Systems Advanced Technology Center \\ Great Baddow, UK
}


second set of "Huygen Snapshots" are recorded for a third stage of small high resolution models to record the fields excited on each antenna element, allowing the calculation of inter-element coupling for the array.

This Three Stage Method has been used to support investigation into the performance a range of different antennas in Conformal Antenna Arrays. A Conformal Antenna Array Variable Geometry Test Rig, shown in Fig 1 has been produced to allow direct comparisons between the array performance for each different antenna type. Array measurements have been completed for two different antenna types, a Cavity Backed Slot antenna Fig 2a, and a Dual Feed Dual Circular Patch Antenna Fig. 2b. However the FDTD predictions for the Dual Patch antenna are not yet completed and so only the Cavity Slot Array will be considered.

This allows the correlation coefficients between modelled and measured array antenna patterns, and Scattering matrix data to be calculated, giving a statistical measure for the accuracy of this method.

\section{RESUlTS}

\section{A. Array Pattern Comparison}

There is good agreement between the modelled and measured antenna array patterns ( 0 degrees Fig. 3a, and 20 degrees Fig. 3b Total Power Pattern shown in x-y plane in line with the Test Rig Long axis). In order to numerically assess the FDTD model performance, the antenna array patterns are subjected to Pearson product-moment correlation analysis, showing only an average correlation of $12.7 \%$.

\section{B. Array Inter-Element Coupling}

The performance analysis between measurements and predictions for $\mathrm{S}$ matrix terms gives some interesting results (Fig 4). All Scattering model and measured pairs have an average correlation of $54.41 \%$. However upon closer inspection it is revealed that when separated into groups of inter-element coupling terms and element $S_{1,1}$ terms, the $S_{n=n}$ pairs all show a strong correlation with a mean of $88.47 \%$.

\section{CONCLUSION}

An assessment of the difference in performance between the Cavity Slot Antenna Array Patterns and the Scattering matrix measurements and predictions suggests to the author that the Three stage method is accurately modelling the individual antenna elements, and it is the area between the elements and the flexible copper ground plane to which they are joined by copper tape and solder.

It is suggested that given a more consistent relationship between element and ground plane that might be more readily modelled the array patterns would show a much higher correlation with the measurements. Given that a conventional approach would have encountered the same difficulties, and incurred a much high cost in terms of computational time, this method has great potential to become a highly attractive tool for Conformal Antenna Array Design.

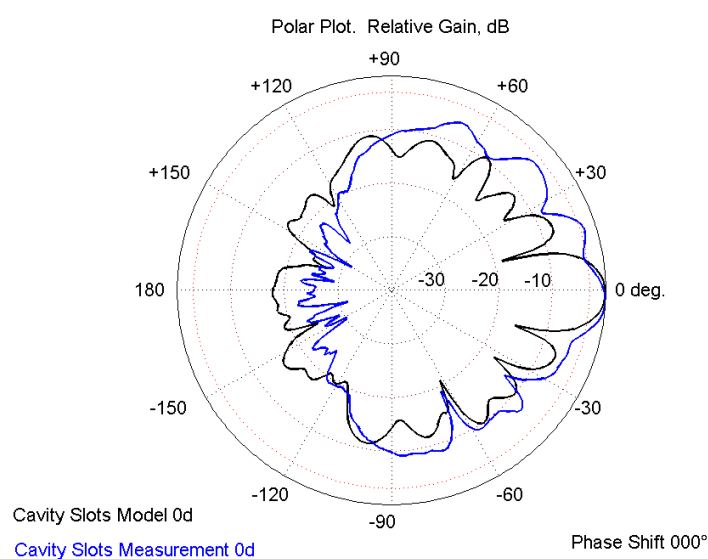

(a)

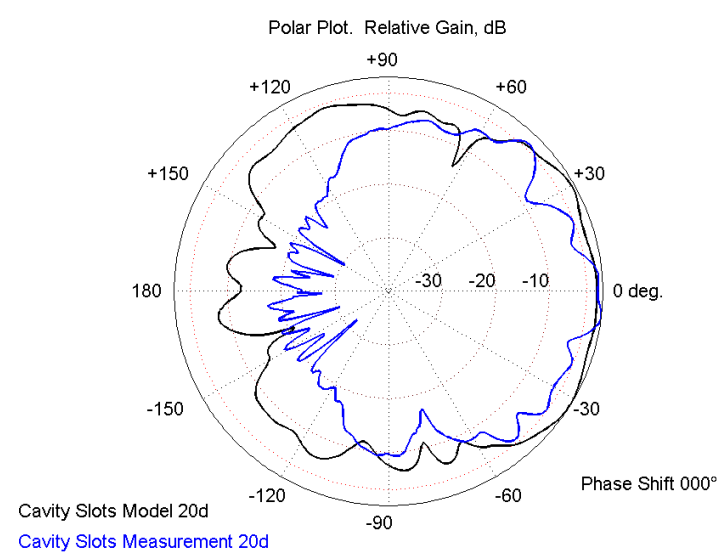

(b)

Fig. 3. Cavity Slot Pattern Comparison (0 degrees) (a) and Cavity Slot Pattern Comparison (20 degrees) (b)

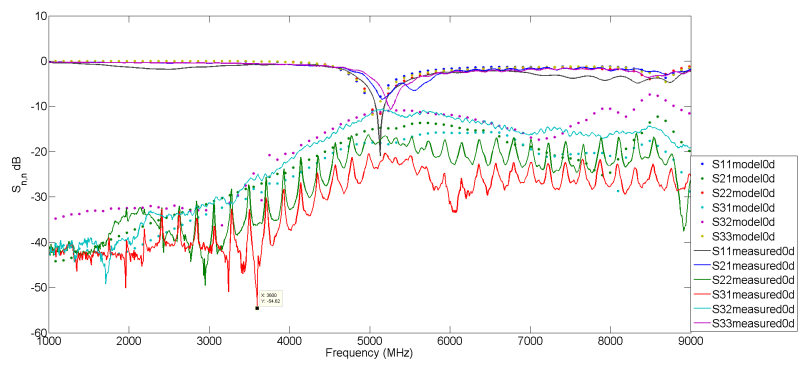

Fig. 4. Comparison of FDTD Predictions and $S_{m, n}$ measurements

\section{REFERENCES}

[1] K. Yee "Numerical Solution of Initial Boundary Value Problems involving Maxwell's Equations in Isotropic Media", IEEE Transactions on Antennas and Propagation, V 14, No 5, May 1966, pp 302-307.

[2] C. Christodoulou, "Analysis of a UWB Hemispherical Antenna Array in FDTD with a Time Domain Huygens Method", IEEE Transactions on Antennas and Propagation, V 60, N 11, November 2012, pp 5251-5258. 